29.

\title{
Bemerkungen zur Integration der Differential- Gleichungen erster Ordnung zwischen zwei Veränderlichen.
}

\author{
(Von Herrn Professor Minding.)
}

(Gelesen in der Sitzung der Akademie der Wissenschaften zu St. Petersburg am 6ten Juni 1845 und aus dem Bulletin tome IV. S. 378 mitgetheilt.)

Unter den Hülfsmitteln, welche man bis jetzt für diese Integration gefunden hat, dürfte die Benutzung particulärer Integrale eines der umfassendsten sein; wenigstens wird durch sie die Lösung der schwierigsten unter den von Euler in den Inst. calc. int. aufgestellten Beispielen wesentlich erleichtert und gewinnt ein mehr methodisches Ansehen. Dahin gehört z. B. das 55te Problem (S. 207 des ersten Bandes), in welchem die Integration der Gleichung $y d y+(a+b x+n x x) d y=y(c+n x) d x$ gefordert und durch eine Substitution geleistet wird, von welcher Euler am Schlusse selbst sagt: „Casu autem hic vix praevidendo evenit, ut haec substitutio ad votum successerit; neque hoc problema magnopere juvabit." Diese Gleichung ist neuerlich von Jacobi im 24ten Bande dieses Journals in erweiterter Gestalt behandelt worden; aber durch Aufsuchung und Benutzung particulärer Integrale-läfst sich ihre Integration noch sebr vereinfachen.

Es seien $\boldsymbol{M}$ und $\boldsymbol{N}$ zwei ganze Polynome in $y$, deren Coëfficienten beliebige Functionen von $x$ sind, und $M d x+N d y=0$ sei die vorgelegte Gleichung. Hat man eine gewisse Anzahl $(\mu)$ particulärer Integrale dieser Gleichung, nämlich $y_{1}, y_{2}, \ldots y_{\mu}$, und sind $M_{\mu}$ und $N_{\mu}$ die Werthe von $M$ und $N$, welche sich durch Einsetzung von $y_{\mu}$ für $y$ ergeben, so ist $M_{1} d x+N_{1} d y_{1}=0$, $M_{2} d x+N_{2} d y=0$, u. s. f. Die Benutzung dieser particulären Integrale giebt nun zu mancherlei Transformationen Gelegenheit, unter welchen ich die folgende hervorhebe. Man bilde das Product $\psi y=\left(y-y_{1}\right)\left(y-y_{2}\right) \ldots\left(y-y_{\mu}\right)$, so erhält man durch Zerlegung in einfache Brüche:

$$
\begin{aligned}
\frac{\boldsymbol{M}}{\psi y} & =\boldsymbol{G}+\frac{\boldsymbol{M}_{1}}{\psi^{\prime} y_{1}\left(y-y_{1}\right)}+\cdots+\frac{\boldsymbol{M}_{\mu}}{\psi^{\prime} y_{\mu}\left(y-y_{\mu}\right)} \\
\frac{\boldsymbol{N}}{\psi y} & =\boldsymbol{H}+\frac{\boldsymbol{N}_{1}}{\psi^{\prime} y_{1}\left(y-y_{1}\right)}+\cdots+\frac{\boldsymbol{N}_{\mu}}{\psi^{\prime} y_{\mu}\left(y^{\prime}-y_{\mu}\right)} .
\end{aligned}
$$

$\boldsymbol{G}$ und $\boldsymbol{H}$ bedeuten die in den Quotienten enthaltenen ganzen Functionen von $\boldsymbol{y}$. 
Bemerkt man noch, dafs $M_{1} d x=-N_{1} d y_{1}$, u. s. f. ist, so ergiebt sich

$$
\frac{M d x+N d y}{\psi y}=\boldsymbol{G} d x+\boldsymbol{H} d y+\frac{N_{1} d\left(y-y_{1}\right)}{\psi^{\prime} y_{1}\left(y-y_{1}\right)}+\cdots+\frac{N_{\mu} d\left(y-y_{\mu}\right)}{\psi^{\prime} y_{\mu}^{\prime}\left(y-y_{\mu}\right)}=0 .
$$

Durch diese Transformation wird die Gleichung

$$
\left(\boldsymbol{A}+\boldsymbol{B} y+C \boldsymbol{y}^{2}\right) d x+d y=0,
$$

wo $\boldsymbol{A}, \boldsymbol{B}, \boldsymbol{C}$ beliebige Functionen von $x$ sind, sofort integrabel, wenn zwei particuläre Integrale derselben gegeben sind. Da nämlich $N=1, \psi y=\left(y-y_{1}\right)\left(y-y_{2}\right)$, $\boldsymbol{H}=\mathbf{0}, \boldsymbol{G}=\boldsymbol{C}$ ist, so verwandelt sich die Gleichung in

oder in

$$
C d x+\frac{d\left(y-y_{1}\right)}{\left(y_{1}-y_{2}\right)\left(y-y_{1}\right)}+\frac{d\left(y-y_{2}\right)}{\left(y_{2}-y_{1}\right)\left(y-y_{2}\right)}=0
$$

$$
C\left(y_{1}-y_{2}\right) d x+\frac{d\left(y-y_{1}\right)}{y-y_{1}}-\frac{d\left(y-y_{2}\right)}{y-y_{2}}=0
$$

wovon $\frac{y-y_{1}}{y-y_{2}} \cdot e^{\int C\left(y_{1}-y_{2}\right) d x}=$ const. das Integral ist.

Die vorstehende Gleichung läfst sich, wie leicht zu sehen, auf die Form $d y+\left(y^{2}+X\right) d x=0$ zurückführen, von welcher Euler (Inst. I. S. 383) zeigt, wie schon aus einem einzigen particulären ihr vollständiges Integral gefunden werden kann. Wenn nämlich der Werth $y_{1}$ von $y$ der Gleichung genügt, so ist $d y+y^{2} d x=d y_{1}+y_{1}^{2} d x$ oder $d\left(y-y_{1}\right)+\left(y-y_{1}\right)\left(y+y_{1}\right) d x=0$. Dividirt man diese Gleichung mit $\left(y-y_{1}\right)^{2}$ und setzt $\frac{1}{y-y_{1}}=\approx$, so erhält man $d z=2 y_{1} z d x+d x$, mithin durch Integration dieser linearen Gleichung:

$$
z=\frac{1}{y-y_{1}}=e^{2 \int y_{1} d x}\left(\text { const. }-\int e^{-2 \int y_{1} d x} d x\right) \text {. }
$$

Aus diesem schönen Eulerschen Resultate kann das obige leicht abgeleitet werden; denn es folgt daraus zuerst $\frac{d\left(y-y_{1}\right)}{y-y_{1}}+\left(y+y_{1}\right) d x=0$, mithin $\left(y-y_{1}\right) e^{\int\left(y+y_{1}\right) d x}=$ const. $=a$; und eben so, wenn $y_{2}$ ein zweites particuläres Integral ist, $\left(y-y_{2}\right) e^{\int\left(y+y_{2}\right) d x}=b$; folglich, wenn $e^{\int y d x}$ durch Division weggeschafft wird, $\frac{y-y_{1}}{y-y_{2}} e^{\int\left(y_{1}-y_{2}\right) d x}=\frac{a}{b}=$ const. Was man daher in diesem Falle durch die angegebene Transformation erlangt, kommt an Werth dem Eulerschen Resultate nicht gleich.

Bezeichnet man durch $M d x+\mathbf{N d y}=\mathbf{0}$ die von Jacobi in diesem Journal behandelte Gleichung, so ist

$$
\begin{gathered}
\boldsymbol{M}=\boldsymbol{C}+\boldsymbol{C}^{\prime} \boldsymbol{x}+\boldsymbol{C}^{\prime \prime} \boldsymbol{y}-\left(\boldsymbol{A}+\boldsymbol{A}^{\prime} \boldsymbol{x}+\boldsymbol{A}^{\prime \prime} y\right) y, \\
\boldsymbol{N}=\left(\boldsymbol{A}+\boldsymbol{A}^{\prime} \boldsymbol{x}+\boldsymbol{A}^{\prime \prime} \boldsymbol{y}\right) \boldsymbol{x}-\left(\boldsymbol{B}+\boldsymbol{B}^{\prime} \boldsymbol{x}+\boldsymbol{B}^{\prime \prime} y\right) .
\end{gathered}
$$


Man genügt dieser Gleichung durch die Annahme $y=\alpha x+\beta$, wenn $\alpha$ und $\beta$ nach folgenden Bedingungen bestimmt werden:

$$
\begin{aligned}
\left(\boldsymbol{A}+\boldsymbol{A}^{\prime \prime} \beta\right) \beta+\left(\boldsymbol{B}+\boldsymbol{B}^{\prime \prime} \beta\right) \alpha & =\boldsymbol{C}+\boldsymbol{C}^{\prime \prime} \boldsymbol{\beta} \\
\left(\boldsymbol{A}^{\prime}+\boldsymbol{A}^{\prime \prime} \alpha\right) \boldsymbol{\beta}+\left(\boldsymbol{B}^{\prime}+\boldsymbol{B}^{\prime \prime} \alpha\right) \alpha & =\boldsymbol{C}^{\prime}+\boldsymbol{C}^{\prime \prime} \alpha .
\end{aligned}
$$

Hieraus erhält man für $\alpha$ eine Gleichung dritten Grades. Es seien $\alpha_{1}, \alpha_{2}, \alpha_{3}$ ihre Wurzeln und $\beta_{1}, \beta_{2}, \beta_{3}$ die entsprechenden Werthe von $\beta$, so hat man drei particuläre Integrale $y_{1}=\alpha_{1} x+\beta_{1}, y_{2}=\alpha_{2} x+\beta_{2}, \quad y_{3}=\alpha_{3} x+\beta_{3}$. Ist wieder $\psi y=\left(y-y_{1}\right)\left(y-y_{2}\right)\left(y-y_{3}\right)$, so erhält man

$$
\frac{M d x+N d y}{\psi y}=\frac{N_{1} d\left(y-y_{1}\right)}{\psi^{\prime} y_{1}\left(y-y_{1}\right)}+\frac{N_{2} d\left(y-y_{2}\right)}{\psi^{\prime} y_{2}\left(y-y_{2}\right)}+\frac{N_{3} d\left(y-y_{3}\right)}{\psi^{\prime} y_{3}\left(y-y_{3}\right)} .
$$

Es läfst sich fast ohne alle Rechnung zeigen, dafs die Quotienten $\frac{N_{1}}{\psi^{\prime} y_{1}}=q_{1}$, $\frac{N_{2}}{\psi^{\prime} y_{2}}=q_{2}, \frac{N_{3}}{\psi^{\prime} y_{3}}=q_{3}$ constant sind. Es ist nämlich

$$
\boldsymbol{N}_{1}=\left(\boldsymbol{A}+\boldsymbol{A}^{\prime} \boldsymbol{x}+\boldsymbol{A}^{\prime \prime} y_{1}\right) \boldsymbol{x}-\left(\boldsymbol{B}+\boldsymbol{B}^{\prime} \boldsymbol{x}+\boldsymbol{B}^{\prime \prime} y\right)=\left(\boldsymbol{A}^{\prime}+\boldsymbol{A}^{\prime \prime} \alpha_{1}\right) \boldsymbol{x}^{2}+\cdots
$$

ein Polynom vom zweiten Grade. Eben so ist auch

$$
\psi^{\prime} y_{1}=\left(y_{1}-y_{2}\right)\left(y_{1}-y_{3}\right)
$$

ein Polynom zweiten Grades. Bezeichnet man den Werth von $x$, für welchen $y_{1}=y_{2}$ ist, durch $x_{3}$, und den Werth von $x$, für welchen $y_{1}=y_{3}$ ist, durch $x_{2}$, so ist

$$
\psi^{\prime} y_{1}=\left(\alpha_{1}-\alpha_{2}\right)\left(\alpha_{1}-\alpha_{3}\right)\left(x-x_{2}\right)\left(x-x_{3}\right) .
$$

Bemerkt man nun, dafs $M_{1}+N_{1} \alpha_{1}\left(=M_{1}+N_{1} \frac{d y_{1}}{d x}\right)$ und eben so $M_{2}+N_{2} \alpha_{2}$ für jeden Werth von $x$ identisch Null sind, und dafs folglich für $x=x_{3}$ und $y_{1}=y_{2}, M_{1}=M_{2}, N_{1}=N_{2}$ werden: so hat man für $x=x_{3}, M_{1}+N_{1} \alpha_{1}=0$, $M_{1}+N_{1} \alpha_{2}=0$, folglich, da im Allgemeinen $\alpha_{1}$ von $\alpha_{2}$ verschieden ist, $\boldsymbol{M}_{1}=0$, $N_{1}=0$; daher ist $N_{1}$ theilbar durch $x-x_{3}$, und eben so durch $x-x_{2}$; folglich $\boldsymbol{N}_{1}=\left(\boldsymbol{A}^{\prime}+\boldsymbol{A}^{\prime \prime} \alpha_{1}\right)\left(x-x_{2}\right)\left(x-x_{3}\right)$, mithin

$$
q_{1}=\frac{A^{\prime}+A^{\prime \prime} \alpha_{1}}{\left(\alpha_{1}-\alpha_{2}\right)\left(\alpha_{1}-\alpha_{3}\right)} \text {. }
$$

Auf gleiche Weise ergeben sich $q_{2}$ und $q_{3}$, und hieraus folgt sofort das Integral der vorgelegten Gleichung, nämlich

$$
\left(y-y_{1}\right)^{q_{1}}\left(y-y_{2}\right)^{q_{2}^{*}}\left(y-y_{3}\right)^{q_{3}}=\text { const. }
$$

wobei noch zu bemerken, dafs $q_{1}+q_{2}+q_{3}=0$, weil $\boldsymbol{N}$ in Bezug auf $\boldsymbol{y}$ vom ersten, $\psi y$ vom dritten Grade und $q_{1}=\frac{N_{1}}{\psi^{\prime} y_{1}}$ u. s. f. ist. 
Sind $\boldsymbol{M}$ und $\boldsymbol{N}$ zwei homogene.Functionen von $x$ und $\boldsymbol{y}$ von gleichen Graden, so dient bekanntlich die Substitution $y=t x$ zur Integration der Gleichung $\boldsymbol{M} d \boldsymbol{d}+\boldsymbol{N} d y=0$. Dieselbe Substitution erstreckt sich auch auf die allgemeinere Gleichung $\boldsymbol{M} d x+\boldsymbol{N} d y+\boldsymbol{Q}(\boldsymbol{x} d y-y d x)=0$, in welcher $\boldsymbol{M}$ und $\boldsymbol{N}$ homogen und von gleichem Grade $\boldsymbol{n}$ sind, $\boldsymbol{Q}$ aber eine beliebige Function von $\frac{y}{x}$ ist. Denn setzt man $y=t x$, so wird $M=x^{n} \boldsymbol{T}, N=x^{n} \boldsymbol{T}_{1}$, wo $T, T_{1}$, so wie $\boldsymbol{Q}$, Functionen von $t$ sind, und die vorgelegte Gleichung verwandelt sich in folgende:

$$
\left(\boldsymbol{T}+t \boldsymbol{T}_{1}\right) x^{n-2} d x+T_{1} x^{n-1} d t+Q d t=0,
$$

welche, weil sie lineär nach $x^{n-1}$ ist, folgendes Integral giebt:

$$
\frac{x^{n-1}}{n-1} \cdot e^{\int \frac{(n-1) T_{1} d t}{T+t T_{1}}}+\int e^{\int \frac{(n-1) T_{1} d t}{T+t T_{1}}} \frac{Q d t}{T+t T_{1}}=\text { const. }
$$

Dieses Verfahren ist auch anwendbar, wenn $\boldsymbol{Q}$ eine homogene Function von $x$ und $y$ von beliebigem Grade $q$ ist; denn für $y=t x$ wird $Q=x^{q} f(t)$ und durch Division mit $x^{q}$ erhält die Gleichung die oben angenommene Form. Man findet hiernach z. B. das Integral der folgenden Gleichung:

$$
\left(a x^{2}+b x y+c y^{2}\right) d x+\left(a^{\prime} x^{2}+b^{\prime} x y+c^{\prime} y^{2}\right) d y+g(y d x-x d y)=0 .
$$

Dieses Integral ist

$$
\begin{gathered}
\left(y-\mu_{1} x\right)^{A_{1}}\left(y-\mu_{2} x\right)^{A_{2}}\left(y-\mu_{3} x\right)^{A_{3}} \\
+\frac{g}{c^{\prime}} \int(y d x-x d y)\left\{\left(y-\mu_{1} x\right)^{A_{1}-1}\left(y-\mu_{2} x\right)^{A_{2}-1}\left(y-\mu_{3} x\right)^{A_{3}-1}\right\}=\text { const. }
\end{gathered}
$$

Die Gröfsen $\mu_{1}, \mu_{2}, \mu_{3}$ sind die Wurzeln der Gleichung

$$
a+b \mu+c \mu^{2}+\left(a^{\prime}+b^{\prime} \mu+c^{\prime} \mu^{2}\right) \mu=0 .
$$

Der Werth von $A_{1}$ ist $A_{1}=\frac{a^{\prime}+b^{\prime} \mu_{1}+c^{\prime} \mu_{1}^{2}}{c^{\prime}\left(\mu_{1}-\mu_{2}\right)\left(\mu_{1}-\mu_{3}\right)}$, und eben so werden $A_{2}$ und $\boldsymbol{A}_{3}$ bestimmt. Ferner ist $\boldsymbol{A}_{1}+\boldsymbol{A}_{2}+\boldsymbol{A}_{3}=1$ und vermöge dieser Bedingung wird der Ausdruck unter dem Integralzeichen ein vollständiges Differential von der Form $\varphi\left(\frac{y}{x}\right) d\left(\frac{y}{x}\right)$.

Unter den von Euler gegebenen Beispielen findet sich S. 347 folgendes:

$$
y d x-x d y+a x^{n} y d y\left(x^{n}+b\right)^{\frac{1}{n}}=0,
$$

zu dessen Integration wieder eine "substitutio non adeo obvia", nämlich

$$
y v=\frac{1}{\left(x^{n}+b\right)^{\frac{1}{n}}}
$$


gebraucht wird. Der Schlüssel zu dieser Substitution liegt in der Bemerkung, dafs

$$
y_{1}=\frac{-x}{a b\left(x^{n}+b\right)^{\frac{1}{n}}}
$$

ein particuläres Integral der Gleichung ist. Drückt man $x$ durch $y_{1}$ aus und eliminirt alsdann $x$ aus der Differentialgleichung, so erhält sie die Form

$$
y_{1} d y-y d y_{1}+k y_{1}^{n}\left(y-y_{1}\right) d y=0 \text {, }
$$

wo $k=(-a b)^{n}$. Diese Gleichung fällt augenscheinlich unter die so eben angegebene Erweiterung der Regel der homogenen Functionen und wird mithin durch die Substitution $y=t y_{1}$, oder auch durch $y_{1}=t y$, integrabel. Die zweite dieser Substitutionen, welche Euler anwendet, ist die bequemere. 